\title{
Does Blood Flow Change according to Mood? Blood Rheology in Bipolar Disorder
}

\author{
Tevfik Kalelioglu', Murat Kocabiyik ${ }^{2}$, Burcu Kok ${ }^{1}$, Pelin Unalan ${ }^{1}$, Sule Sozen ${ }^{1}$, Ozge Yuksel ${ }^{1}$, \\ Nesrin Karamustafalioglu ${ }^{1}$ \\ ${ }^{1}$ Department of Psychiatry, Bakırköy Mental Health Research and Teaching Hospital, Istanbul, ${ }^{2}$ Department of Biochemistry, Bingöl State \\ Hospital, Bingöl, Turkey
}

\begin{abstract}
Objective: Bipolar disorder (BD) is associated with increased rates of cardiovascular diseases. There is growing evidence that blood viscosity may have a common role, correlated with well-known major risk factors that promote cardiovascular disease. In this study we aimed to investigate the whole blood viscosity (WBV) in different stages of BD.

Methods: A total of 121 bipolar patients and 41 age-gender matched healthy controls were included. Forty-four of bipolar patients were in manic, 35 were depressed and 42 were in euthymic state. WBV was calculated from hematocrit and total plasma protein according to Simone's formula at low and high shear rates (LSR and HSR).

Results: WBV at HSR of manic group was 16.91 \pm 1.01 , depressive group was $17.23 \pm 0.80$, euthymic group was $17.63 \pm$ 0.95, and control group was 17.52 $\pm 0.71(p=0.001)$. WBV at LSR of manic depressive, euthymic and control group were $53.10 \pm 20.58,60.30 \pm 17.02,68.91 \pm 20.33$, and $62.01 \pm 19.28$, respectively $(p=0.001)$. Both WBV at HSR and LSR of manic group was significantly lower than that of the euthymic and control groups $(p=0.001$ and 0.010 respectively for HSR, $p=0.001$ and 0.011 respectively for LSR). WBV was significantly positively correlated with lipid profile except high density lipoprotein (HDL).

Conclusion: Our results demonstrate a decrement in blood viscosity in manic episode compared with euthymics and controls. Positive correlation of blood viscosity with lipid parameters (except HDL), and negative correlation with number of previous manic episodes suggest that manic episode has favorable effect on cardiovascular risk regarding to blood viscosity.
\end{abstract}

KEY WORDS: Blood viscosity; Bipolar disorder; Cardiovascular risk.

\section{INTRODUCTION}

Bipolar disorder (BD) is associated with an increased risk of cardiovascular diseases (CVD) which is one of the leading cause of shorter life expectancy than general population. ${ }^{1)}$ In addition to well known risk factors for CVD, there is a growing evidence that blood viscosity is associated with increased cardiovascular risk. ${ }^{2)}$

Basically, viscosity can be defined as stickiness and thickness of blood. Internal friction that develops between

Received: June 20, 2017 / Revised: September 2, 2017

Accepted: September 26, 2017

Address for correspondence: Tevfik Kalelioglu, MD

Department of Psychiatry, Bakırköy Mental Health Research and Teaching Hospital, Zuhuratbaba Mah. Dr.Tevfik Sağlam

Cad. No:25/2 , Istanbul 34147, Turkey

Tel: +90-212-543-6565, Fax: +90-212-409-1595

E-mail: tevfikkaleli@hotmail.com

ORCID: https://orcid.org/0000-0001-9319-055X adjacent layers of flowing blood causes an intrinsic resistance which is also called as viscosity. The velocity gradient during blood flow is called the shear rate. Viscosity is relatively high at low shear rates (LSR), as when blood is moving at a low velocity during diastole. At high shear rates (HSR), as during systole, viscosity relatively decreases. ${ }^{3)}$ Major risk factors for CVD such as hypertension, hyperlipidemia, obesity, cigarette smoking, male gender, and aging have been correlated with whole blood viscosity (WBV). It has been suggested that WBV may have an integrated role with other conventional risk factors in the mechanism of developing CVD. ${ }^{4)}$

In previous studies, both physical and emotional stress have been shown to cause changes in hemorheologic measures such as hematocrit $(\mathrm{HCT})$, hemoglobin, total plasma protein (TP) concentrations, whole blood and plasma viscosity. ${ }^{5,6)}$ Early studies of hemorheologic meas-

(c) This is an Open-Access article distributed under the terms of the Creative Commons Attribution Non-Commercial License (http://creativecommons.org/licenses/by-nc/4.0) which permits unrestricted non-commercial use, distribution, and reproduction in any medium, provided the original work is properly cited. 
ures among patients diagnosed with BD have shown that although hematologic parameters remained in the normal range during acute mood exacerbations, mood episodes are associated with a redistribution of body fluids. Mania was found to be associated with a relative fluid retention and hemodilution, while depression is associated with a relative hemoconcentration. ${ }^{7,8)}$

In our study we evaluated the WBV at LSR and HSR with Simone's formula. According to this formula, WBV is calculated from HCT and TP for LSR as "WBV (0.5 sec$1)=(1.89 \times \mathrm{HCT})+3.76(\mathrm{TP}-78.42)$ " and HSR as "WBV $(208 \mathrm{sec}-1)=(0.12 \times \mathrm{HCT})+0.17(\mathrm{TP}-2.07)$ ". $\left.{ }^{9}\right)$ Although individuals with BD suffer from cardiovascular morbidity and WBV has a potential to reflect this relationship, there is no clinical study about the WBV in patients with BD. In the current study, for the first time we explored the WBV in manic, depressive and euthymic states of BD and compared with healthy controls. Higher manifestation of cardiovascular mortality in BD has been documented before the use of psychotropic medication, suggesting that also the illness itself contributes cardiovascular risk. ${ }^{10)}$ Therefore, we hypothesized that the blood viscosity which has a potential to reflect the cardiovascular risk status, should be higher in BD patient groups than healthy controls.

\section{METHODS}

\section{Participants}

BD in-patients with mania in the current episode, hospitalized or outpatients with BD depression and euthymic outpatients according to the Diagnostic and Statistical Manual of Mental Disorders 5th edition (DSM-5) criterion, were screened for the study. Severity of mania was evaluated with the Young Mania Rating Scale (YMRS). Bipolar depression was assessed with the 17-Item Hamilton Depression Rating Scale (HAM-D); a severity score of 20 or greater was required for inclusion. Euthymic state was defined as YMRS $\leq 7$ and HAM-D score $\leq 7 .{ }^{11)}$ Diagnosis of patient groups was established by a consensus of two senior psychiatrists following a psychiatric interview according to DSM-5. Additionally age, gender and smoking status matched healthy controls were enrolled in the study. Healthy controls were selected between hospital employees with no previous history of psychiatric disorder, mental retardation, alcohol and/or substance abuse and presence of acute infectious disorder and renal dysfunction. All groups were composed of male individuals between the ages of 18-65 years. Exclusion criteria were any co-morbid psychiatric disorder other than BD, mental retardation, alcohol and/or substance abuse, presence of acute infectious disorder and renal dysfunction. Blood samples were drawn in the morning at around 8 a.m. from a forearm vein at the end of an overnight fasting period for at least 8 hours to obtain standardization for water-food intake. Routine biochemical evaluation and hemogram tests were performed for all participants. This study protocol was approved by local ethical committee at Bakırköy Mental Health Research and Training State Hospital (No. 02.05.2017/20). All participants and/or their legal representatives provided written informed consent before participating in the study.

\section{Statistics}

The categorical variables were presented as percentages and continuous variables were reported as mean \pm standard deviation. Chi-square test was used for comparison of categorical variables. The normality of the distribution was assessed by the Kolmogorov-Smirnov test. In comparing the continuous variables between groups, Kruskal-Wallis test for nonparametric and analysis of variance (ANOVA) with Tukey's post-hoc analysis for parametric values, were used. For pairwise group comparison of nonparametric continuous variables, Bonferroni corrected Mann Whitney $U$ test was performed. Spearman's correlation test was used to determine the linear associations between variables. The level of significant difference was regarded as $p<0.05$. Statistical analysis was performed using Statistical Package for Social Sciences (SPSS) version 22 for Windows (IBM Co., Armonk, NY, USA).

\section{RESULTS}

Forty-four manic, 35 depressed, 42 euthymic BD patients and 41 healthy age and gender matched controls were included in the study. All groups were consisted of male individuals. There was no statistically significant difference according to mean age between groups (38.22 \pm 11.37 years for manic, $41.54 \pm 10.29$ years for depressed, $40.52 \pm 10.90$ years for euthymic, and $39.02 \pm 10.69$ years for controls; $p=0.530)$. Statistically significant difference was observed between manic (11.65 \pm 9.42 years), depressive ( $17.54 \pm 9.55$ years) and euthymic ( $14.96 \pm 9.18$ years $)$ 
groups in terms of illness duration $(p=0.009)$ (pairwise comparisons; manic vs. depressive [ $p=0.009]$, manic vs. euthymic $[p=0.156]$, depressive vs. euthymic $[p=0.597]$ ). The average YMRS scores of manic and euthymic patients were $37.38 \pm 7.21$ and $0.09 \pm 0.3$ respectively, and HAM-D scores of depressed and euthymic patients were $41.74 \pm$ 4.55 and $0.26 \pm 0.66$ respectively. The smoking status and body mass index (BMI) were not statistically different between groups $\left(\chi^{2}=4.56, p=0.207\right.$ and 0.503 respectively). Regarding lipid profile of manic, depressive, euthymic and control groups; total cholesterol levels were $163.77 \pm$ $27.97 \mathrm{mg} / \mathrm{dl}, 188.88 \pm 57.87 \mathrm{mg} / \mathrm{dl}, 186.54 \pm 43.91 \mathrm{mg} / \mathrm{dl}$, $199.78 \pm 38.24 \mathrm{mg} / \mathrm{dl}$ respectively ( $p<0.001$ ); low density lipoprotein (LDL) cholesterol levels were 91.78 \pm 27.63 $\mathrm{mg} / \mathrm{dl}, 111.92 \pm 60.01 \mathrm{mg} / \mathrm{dl}, 109.49 \pm 39.83 \mathrm{mg} / \mathrm{dl}$, and $127.73 \pm 34.70 \mathrm{mg} / \mathrm{dl}$ respectively $(p<0.001)$; high density lipoprotein (HDL) cholesterol levels were $43.38 \pm$ $12.38 \mathrm{mg} / \mathrm{dl}, 38.25 \pm 11.04 \mathrm{mg} / \mathrm{dl}, 44.78 \pm 12.52 \mathrm{mg} / \mathrm{dl}$, and $43.97 \pm 11 \mathrm{mg} / \mathrm{dl}$ respectively ( $p=0.054)$; and triglyceride levels were $141.15 \pm 94.84 \mathrm{mg} / \mathrm{dl}, 217.17 \pm 159.68$ $\mathrm{mg} / \mathrm{dl}, 160.92 \pm 99.96 \mathrm{mg} / \mathrm{dl}$, and $138.14 \pm 66.18 \mathrm{mg} / \mathrm{dl}$ respectively $(p=0.014)$ (Table 1 ).

Except 3 patients, manic group was medication free (of two were under treatment with valproate and 1 with lithium). Depressed patients were medicated with antipsychotics $(94.3 \%)$, lithium (62.9\%), antidepressants $(37.1 \%)$, valproat $(28.6 \%)$, lamotrigine $(17.1 \%)$, and karbamazepine $(2.9 \%)$. Euthymic patients were under treatment with antipsychotics $(76.2 \%)$, lithium $(73.8 \%)$, val- proat $(31 \%)$, carbamazepine $(2.4 \%)$, and lamotrigine (2.4\%). In terms of comorbid medical illness, 2 of manic ( 1 thyroid disease, 1 hypertension), 9 of depressive ( 3 thyroid disease, 5 diabetes, 1 hypertension), 3 of euthymic ( 1 thyroid disease, 1 hypertension, 1 diabetes), and 4 of controls ( 3 diabetes, 1 thyroid disease) were under medical treatment. None of participants had previous history of CVD.

When we compare the components of WBV, there was no statistically significant difference between groups in terms of HCT $(44.35 \pm 3.67 \%$ for manic, $44.24 \pm 2.74 \%$ for depressed, $44.53 \pm 2.93 \%$ for euthymic, and $45.64 \pm 3.55 \%$ for controls, $p=0.199)$. Total protein level of manic group $(70.24 \pm 4.60 \mathrm{~g} / \mathrm{L})$ was statistically significantly lower than that of the euthymic $(74.36 \pm 5.07 \mathrm{~g} / \mathrm{L})$ and control $(72.94 \pm 3.68 \mathrm{~g} / \mathrm{L})$ groups $(p<0.001$ and $p=0.031$ respectively). However, there was no significant statistical difference between depressive patients $(72.19 \pm 4.24 \mathrm{~g} / \mathrm{L})$ and none of other groups regarding to total protein levels. HCT and total protein levels are shown in Table 2.

In respect of viscosity, WBV at HSR value of manic group was $16.91 \pm 1.01$, depressive group was $17.23 \pm$ 0.80 , euthymic group was $17.63 \pm 0.95$, and control group was $17.52 \pm 0.71(p=0.001)$. Regarding WBV at LSR values, also statistically significant difference was observed between groups $(p=0.001)$. LSR values of manic depressive, euthymic and control group were $53.10 \pm 20.58$, $60.30 \pm 17.02,68.91 \pm 20.33$, and $62.01 \pm 19.28$ respectively (Table 2). After adjustment for age, smoking, BMI,

Table 1. Characteristics and lipid profile of groups

\begin{tabular}{|c|c|c|c|c|c|}
\hline Characteristic & Manic & Depressive & Euthymic & Control & $p$ value \\
\hline Age $(y r)^{\mathrm{a}}$ & $38.22 \pm 11.37$ & $41.54 \pm 10.29$ & $40.52 \pm 10.90$ & $39.02 \pm 10.69$ & 0.530 \\
\hline Duration of illness $(y r)^{b}$ & $11.65 \pm 9.42$ & $17.54 \pm 9.55$ & $14.96 \pm 9.18$ & - & $0.009^{* *}$ \\
\hline YMRS score & $37.38 \pm 7.21$ & - & $0.09 \pm 0.3$ & - & $<0.001^{* *}$ \\
\hline HAM-D score & - & $41.74 \pm 4.55$ & $0.26 \pm 0.66$ & - & $<0.001^{* *}$ \\
\hline Previous manic episodes & $5.55 \pm 4.59$ & $3.87 \pm 3.80$ & $4.59 \pm 3.60$ & - & \\
\hline Previous depressive episodes & $1.68 \pm 1.39$ & $4.88 \pm 3.66$ & $2.06 \pm 1.65$ & - & \\
\hline BMI $\left(\mathrm{kg} / \mathrm{m}^{2}\right)^{\mathrm{b}}$ & $27.59 \pm 6.58$ & $27.56 \pm 4.06$ & $27.28 \pm 5.14$ & $26.03 \pm 3.47$ & 0.503 \\
\hline Smoking status (yes) ${ }^{\mathrm{c}}$ & $70.4 \%$ & $68.5 \%$ & $50.0 \%$ & $63.4 \%$ & 0.207 \\
\hline Total cholesterol $(\mathrm{mg} / \mathrm{dl})^{\mathrm{b}}$ & $163.77 \pm 27.97$ & $188.88 \pm 57.87$ & $186.54 \pm 43.91$ & $199.78 \pm 38.24$ & $<0.001^{* *}$ \\
\hline $\operatorname{LDL}(\mathrm{mg} / \mathrm{dl})^{\mathrm{b}}$ & $91.78 \pm 27.63$ & $111.92 \pm 60.01$ & $109.49 \pm 39.83$ & $127.73 \pm 34.70$ & $<0.001^{* *}$ \\
\hline $\mathrm{HDL}(\mathrm{mg} / \mathrm{dl})^{\mathrm{b}}$ & $43.38 \pm 12.38$ & $38.25 \pm 11.04$ & $44.78 \pm 12.52$ & $43.97 \pm 11$ & 0.054 \\
\hline Triglyceride $(\mathrm{mg} / \mathrm{dl})^{\mathrm{b}}$ & $141.15 \pm 94.84$ & $217.17 \pm 159.68$ & $160.92 \pm 99.96$ & $138.14 \pm 66.18$ & $<0.014^{*}$ \\
\hline
\end{tabular}

Values are presented as mean \pm standard deviation or percent only.

YMRS, Young Mania Rating Scale; HAM-D, Hamilton Depression Rating Scale; BMI, body mass index; LDL, low density lipoprotein; HDL, high density lipoprotein.

${ }^{\mathrm{a}}$ One-way ANOVA, ${ }^{\mathrm{b}}$ Kruskal-Wallis test, and ${ }^{\mathrm{c}}$ chi-squared test were used; ${ }^{*} p<0.05,{ }^{* *} p<0.01$. 
Table 2. Comparison of whole blood viscosity (WBV) and its components

\begin{tabular}{llllcc}
\hline \multicolumn{1}{c}{ Variable } & Manic & Depressive & Euthymic & Controls & $p$ value \\
\hline WBV at LSR & $53.10 \pm 20.58$ & $60.30 \pm 17.02$ & $68.91 \pm 20.33$ & $62.01 \pm 19.28$ & $0.001^{*}$ \\
WBV at HSR & $16.91 \pm 1.01$ & $17.23 \pm 0.80$ & $17.63 \pm 0.95$ & $17.52 \pm 0.71$ & $0.001^{*}$ \\
Total protein $(\mathrm{g} / \mathrm{L})$ & $70.24 \pm 4.60$ & $72.19 \pm 4.24$ & $74.36 \pm 5.07$ & $72.94 \pm 3.68$ & $<0.001^{*}$ \\
HCT $(\%)$ & $44.35 \pm 3.67$ & $44.24 \pm 2.74$ & $44.53 \pm 2.93$ & $45.64 \pm 3.55$ & 0.199 \\
\hline
\end{tabular}

Values are presented as mean \pm standard deviation.

LSR, low shear rate; HSR, high shear rate; HCT, hematocrit.

One-way ANOVA test was used; ${ }^{*} p<0.01$.

Table 3. Pairwise comparisons ( $p$ values) of WBV at LSR and HSR

\begin{tabular}{lcccccl}
\hline & WBV at LSR & WBV at HSR & $\mathrm{TC}^{\mathrm{b}}$ & $\mathrm{LDL}^{\mathrm{b}}$ & $\mathrm{HDL}^{\mathrm{b}}$ & $\mathrm{TG}^{\mathrm{b}}$ \\
\hline Manic vs. Depressive & 1.000 & 1.000 & $0.046^{*}$ & 0.232 & $0.037^{*}$ & $0.003^{* *}$ \\
Manic vs. Euthymic & $0.014^{*}$ & $0.024^{*}$ & 0.724 & 0.404 & $0.016^{*}$ & 0.063 \\
Manic vs. Control & 0.381 & 0.376 & 0.076 & $0.003^{* *}$ & $0.019^{*}$ & $0.005^{* *}$ \\
Depressive vs. Euthymic & 0.078 & 0.115 & $0.006^{* *}$ & $0.029^{*}$ & 0.647 & 0.304 \\
Depressive vs. Control & 1.000 & 0.964 & $<0.001^{* *}$ & $<0.001^{* *}$ & 0.819 & 0.604 \\
Euthymic vs. Control & 1.000 & 1.000 & 0.155 & $0.025^{*}$ & 0.859 & 0.597 \\
\hline
\end{tabular}

WBV, whole blood viscosity; LSR, low shear rate; HSR, high shear rate; TC, total cholesterol; LDL, low density lipoprotein; HDL, high density lipoprotein; TG, triglyceride; BMI, body mass index.

${ }^{a}$ ANCOVA (analysis of covariance) test results adjusted for age, smoking, BMI, hypertension, cholesterol, trygliceride, and drug medication with Bonferroni's post-hoc analysis and ${ }^{\mathrm{b}}$ Mann-Whitney $U$ tests were used; ${ }^{*} p<0.05,{ }^{* *} p<0.01$.

Table 4. Correlations between whole blood viscosity (WBV) and lipid profile, previous episodes

\begin{tabular}{|c|c|c|c|c|c|c|}
\hline & Total cholesterol & LDL & $\mathrm{HDL}$ & Triglyceride & $\begin{array}{c}\text { Number of } \\
\text { manic episodes }\end{array}$ & $\begin{array}{c}\text { Number of } \\
\text { depressive episodes }\end{array}$ \\
\hline \multirow[t]{2}{*}{ WBV at HSR } & $\mathrm{r}$ ho $=0.320$ & $r$ ho $=0.167$ & $\mathrm{rho}=-0.092$ & $\mathrm{rho}=0.282$ & $\mathrm{rho}=-0.406$ & $\mathrm{rho}=-0.118$ \\
\hline & $p<0.001^{* *}$ & $p=0.036^{*}$ & $p=0.248$ & $p<0.001^{* *}$ & $p<0.001^{* *}$ & $p=0.282$ \\
\hline \multirow[t]{2}{*}{ WBV at LSR } & $\mathrm{r}$ ho $=0.310$ & $\mathrm{rho}=0.157$ & $\mathrm{rho}=-0.091$ & $\mathrm{rho}=0.283$ & $\mathrm{rho}=-0.404$ & $\mathrm{rho}=-0.126$ \\
\hline & $p<0.001^{* *}$ & $p=0.049^{*}$ & $p=0.249$ & $p<0.001^{* *}$ & $p<0.001^{* *}$ & $p=0.252$ \\
\hline
\end{tabular}

LDL, low density lipoprotein; HDL, high density lipoprotein; HSR, high shear rate; LSR, low shear rate.

Spearman's correlation test was used; ${ }^{*} p<0.05,{ }^{* *} p<0.01$.

hypertension, cholesterol, trygliceride, and drug medication with ANCOVA mean values of manic, depressive, euthymic and control groups were as follows: for HSR $17.08 \pm 0.84,17.15 \pm 0.83,17.61 \pm 0.80$, and $17.43 \pm 0.83$ respectively ( $p=0.017)$; for LSR 56.82 $\pm 17.06,58.36 \pm 17.12$, $68.31 \pm 16.46$, and $63.99 \pm 16.97$ respectively $(p=0.011)$, pairwise comparisons of WBV at LSR and HSR are presented in Table 3.

There were statistically significant correlations between WBV and lipid parameters except HDL. When we evaluate the number of previous episode types, we observed a negative, moderate and statistically significant correlation between number of previous manic episodes and WBV in individuals with BD (Table 4).

\section{DISCUSSION}

Adult individuals with BD have a 5-fold increased risk of CVD and manifest CVD 14 years earlier than the population without mood disorders. The traditional cardiovascular risk factors associated with BD are obesity, metabolic syndrome, insulin resistance, diabetes, dyslipidemia, hypertension. ${ }^{10)}$ WBV has been independently correlated with major CVD risk factors. Previous studies suggest that elevated WBV contribute to the risk of developing CVD and may be potentially valuable and useful routine profiling data for cardiovascular risk stratification. $^{3,4)}$

There is a limited number of studies evaluating blood rheology in psychiatric disorders. Effect of psychological 
stress on hemoconcentration has been studied previously. In patients with panic disorder, pentagastrin induced panic symptoms has been found in relation with decreased plasma volume and increased hemoglobin and HCT levels that resulting acute stress-hemoconcentration. ${ }^{12)}$ In a randomized clinical trial conducted by Wong et al., ${ }^{13)}$ WBV was found to be higher in individuals with unipolar depression (UD) when compared with controls. After 8 weeks of antidepressant treatment, hemorheological parameters of stress-hemoconcentration has improved in UD patient group. Abovementioned studies indicate that blood rheology is affected in emotional stress conditions such as psychiatric disorders in both short and long term.

To our knowledge this is the first study in the literature evaluating blood viscosity in BD. The major findings of our study are i) decreased blood viscosity in manic patients compared with euthymic group; ii) concordant with the first finding, there was negative correlation between blood viscosity and number of previous manic episodes in BD subjects; and iii) blood viscosity showed a positive correlation with total cholesterol, LDL and triglyceride levels supporting that blood viscosity may have a common role in cardiovascular morbidity with major risk factors such as lipid profile.

Although WBV at LSR and HSR were highest in euthymic group no statistically significant difference was observed between euthymic-depressive and euthymic-control groups. Interestingly, in contrast to our hypothesis viscosity was significantly lowest in manic group. When we investigated the literature about cardiovascular risk factors at different stages of $\mathrm{BD}$, we found that the risk of CVD was similar to that of our findings in manic episode. Wysokiński et al. ${ }^{14)}$ have compared lipid profile of $\mathrm{BD}$ manic, BD depressive, unipolar depressive, and schizophrenic patients. In terms of total cholesterol, LDL and triglycerides; manic group showed the lowest values. Likewise, in another study, manic patients had significantly lowest cholesterol levels when compared with unipolar or bipolar depressive episode, schizophrenia, schizoaffective disorder, atypical psychosis, and controls. ${ }^{15)}$ Atmaca et al. ${ }^{16)}$ also demonstrated low cholesterol levels in manic group when compared with euthymic and control groups. Taken together, similar to our finding, the abovementioned studies suggest a diminished cardiovascular risk in manic stage.

Imbalance of fluid homeostasis may be another ex- planation of decreased blood viscosity in manic state. Previous case reports have indicated that mania is associated with pathological variations in body weight, retention of water, and accompanied by pathological thirst. ${ }^{17)}$ In a longitudinal study conducted by Hochman et al., ${ }^{7)} \mathrm{HCT}$, hemoglobin, and albumin values were used as indirect measures of hemodilution/hemoconcentration. The authors have reported that during manic episodes, the mean hemoglobin, albumin, and sodium concentrations and HCT were lower than in depressive episodes, suggesting that manic state is characterized by a relative hemodilution. Another study supporting causal relationship between and mood and fluid imbalance demonstrates that mania is associated with an increased risk of lower limb edema. Total protein levels of manic group were significantly lowest in this study. ${ }^{18)}$ In a study by Mert and Terzi ${ }^{8)}$ hematologic parameters such as hemoglobin, $\mathrm{HCT}$ levels in manic episode was also found to be lower than controls. Taken together, previous studies have indicated that manic episode have been associated with decreased levels of hematologic and biochemical parameters which may reflect a relative hemodilution. Decreased blood viscosity of manic patients in our study may be associated with such a hemodilution.

In our study manic group had more previous manic episodes and depressive group had more previous depressive episodes. The consept of "Predominant Polarity" may be an explanation for increased number of previous manic episodes in manic group and also increased depressive episodes in depressive group. ${ }^{19)}$ Negative correlation between blood viscosity and previous number of manic episodes is also interesting. Further studies evaluating the effect of "Predominant Polarity" on blood viscosity are needed to discuss our finding. There was no correlation between blood viscosity and previous depressive episodes.

Limitations of our study are as follows; we hypothesized that BD subjects should have increased blood viscosity reflecting high cardiovascular risk. Although euthymic patients have the highest level of WBV, relatively small sample size might not be adequate for statistically significant results. Blood viscosity has several components. Parameters effecting blood viscosity may interact with each other to provide a balanced homeostasis. ${ }^{20)}$ Therefore confirming our results with viscometer measurement would strengthen our results despite the fact that 
Simone's formula was previously studied and confirmed. In conclusion, we have demonstrated a decrement of blood viscosity in manic episode. In contrast to our hypothesis, although WBV was not useful to reflect a hypothetical increase in cardiovascular risk in bipolar subjects; surprisingly, in concordance with lipid parameters, WBV also has shown a favorable effect of manic episode. Positive correlation of blood viscosity with lipid parameters (except HDL), and negative correlation with number of previous manic episodes indicate that manic episode has favorable effect on cardiovascular risk in terms of blood viscosity.

\section{REFERENCES}

1. Sylvia LG, Shelton RC, Kemp DE, Bernstein EE, Friedman ES, Brody BD, et al. Medical burden in bipolar disorder: findings from the Clinical and Health Outcomes Initiative in Comparative Effectiveness for Bipolar Disorder study (Bipolar CHOICE). Bipolar Disord 2015;17:212-223.

2. Peters SA, Woodward M, Rumley A, Tunstall-Pedoe HD, Lowe GD. Plasma and blood viscosity in the prediction of cardiovascular disease and mortality in the Scottish Heart Health Extended Cohort Study. Eur J Prev Cardiol 2017;24:161-167.

3. Kensey KR. The mechanistic relationships between hemorheological characteristics and cardiovascular disease. Curr Med Res Opin 2003;19:587-596.

4. Cowan AQ, Cho DJ, Rosenson RS. Importance of blood rheo/ogy in the pathophysiology of atherothrombosis. CardiovasC Drugs Ther 2012;26:339-348.

5. Allen MT, Patterson SM. Hemoconcentration and stress: a review of physiological mechanisms and relevance for cardiovascular disease risk. Biol Psychol 1995;41:1-27.

6. Patterson SM, Marsland AL, Manuck SB, Kameneva M, Muldoon MF. Acute hemoconcentration during psychological stress: assessment of hemorheologic factors. Int J Behav Med 1998;5:204-212.

7. Hochman E, Weizman A, Valevski A, Fischel T, Krivoy A. Association between bipolar episodes and fluid and electrolyte homeostasis: a retrospective longitudinal study. Bipolar Disord 2014;16:781-789.

8. Mert DG, Terzi H. Mean platelet volume in bipolar disorder: the search for an ideal biomarker. Neuropsychiatr Dis Treat 2016;12:2057-2062.

9. Cetin MS, Ozcan Cetin EH, Balcı KG, Aydin S, Ediboglu E, et al. The association between whole blood viscosity and coronary collateral circulation in patients with chronic total occlusion. Korean Circ J 2016;46:784-790.

10. Goldstein BI, Carnethon MR, Matthews KA, Mclntyre RS, Miller GE, Raghuveer G, et al. Major depressive disorder and bipolar disorder predispose youth to accelerated atherosclerosis and early cardiovascular disease: a scientific statement from the American heart association. Circulation 2015; 132:965-986.

11. Gubert C, Jacintho Moritz CE, Vasconcelos-Moreno MP, Quadros Dos Santos BT, Sartori J, Fijtman A, et al. Peripheral adenosine levels in euthymic patients with bipolar disorder. Psychiatry Res 2016;246:421-426.

12. Le Melledo JM, Perez-Parada J, Morrow J, Bellavance F, Lara $\mathrm{N}$, Jahandar F, et al. Pentagastrin-induced hemoconcentration in healthy volunteers and patients with panic disorder: effect of pretreatment with ethinyl estradiol. I Psychopharmacol 2011;25:71-77.

13. Wong ML, Dong C, Esposito K, Thakur S, Liu W, Elashoff RM, et al. Elevated stress-hemoconcentration in major depression is normalized by antidepressant treatment: secondary analysis from a randomized, double-blind clinical trial and relevance to cardiovascular disease risk. PLoS One 2008;3:e2350.

14. Wysokiński A, Strzelecki D, Kłoszewska I. Levels of triglycerides, cholesterol, LDL, HDL and glucose in patients with schizophrenia, unipolar depression and bipolar disorder. Diabetes Metab Syndr 2015;9:168-176.

15. Swartz CM. Albumin decrement in depression and cholesterol decrement in mania. J Affect Disord 1990;19:173-176.

16. Atmaca M, Kuloglu M, Tezcan E, Ustundag B, Bayik Y. Serum leptin and cholesterol levels in patients with bipolar disorder. Neuropsychobiology 2002;46:176-179.

17. Crammer JL. Disturbance of water and sodium in a manic-depressive illness. Br J Psychiatry 1986;149:337-345.

18. Hochman E, Krivoy A, Shoval G, Valevski A, Weizman A, Fischel T. Acute manic episode is associated with an increased risk of lower limb edema. Prog Neuropsychopharmacol Biol Psychiatry 2013;47:99-103.

19. Carvalho AF, Mclntyre RS, Dimelis D, Gonda X, Berk M, Nunes-Neto PR, et al. Predominant polarity as a course specifier for bipolar disorder: a systematic review. J Affect Disord 2014; 163:56-64.

20. Cetin EH, Cetin MS, Canpolat U, Aydin S, Aras D, Topaloglu $\mathrm{S}$, et al. Prognostic significance of whole blood viscosity estimated by de Simone's formula in ST-elevation myocardial infarction. Biomark Med 2016;10:495-511. 